\title{
UC 安全的并行可否认认证新方法
}

冯涛 ${ }^{\mathbb{Q} \mathbb{O}^{*}}$,李风华 ${ }^{\circledR}$,马建峰 ${ }^{\oplus}$, 文相在 ${ }^{\circledR}$

(1) 西安电子科技大学 计算机网络与信息安全教育部重点实验室, 西安 710071;

(2) 兰州理工大学 计算机与通信学院，兰州 730050;

(3) 国立庆北大学 移动网络安全技术研究中心, 大邱广域市 702-701, 韩国

* E-mail: fengt@1ut.cn

收稿日期: 2007-05-29; 接受日期: 2007-12-11

国家自然科学基金(批准号：60702059,60633020,60573036)和韩国 MIC 以及 ITRC(批准号：IITA-2006-C1090-0603-0026) 资助项目

摘要 可否认认证协议允许认证者向接收者认证某个消息，但是 接收者不能向第三方证明该认证消息的来源。在考虑开放的异步多 方通信网络环境和自适应的主动攻击者能力的情形下，基于 UC(universally composable)安全模型提出了解决并行可否认认证问 题的新方法. 根据可否认认证协议的安全目标, 定义了形式化的并行 可否认认证理想函数 $F_{\mathrm{CDA}}$, 然后, 利用可验证平滑投影散列函数构 造了一个具体的协议方案, 在公共参考串模型中, 新的协议方案是可 证明UC安全的, 即新方法能够保证可否认认证协议的不同实例在并 行复合情形下是安全的, 当与其他协议同时运行时具有非延展性. 为 了实现可否认认证的前向可否认性, 新方法基于陷门承诺构造了新 的投影密钥函数和可验证平滑投影散列函数，基于证人不可区分，协 议的安全性可以归约为确定性复合剩余假设, 改善了协议的计算效 率和通信效率。

\section{关键词}

信息安全

并行可否认认证

UC 安全模型

可验证平滑投影散列

确定性复合剩余假设

在研究并行零知识证明协议时, Dwork等 ${ }^{[1]}$ 提出了并行可否认认证(concurrent deniable authentication, 简称 CDA). 可否认认证协议是一个两方的通信协议, 该协议代替了非交互的数 字签名机制，能够使接收者 $R$ 确信认证者 $T$ 想要对消息 $m$ 认证，但是接收者 $R$ 不能向第 3 方证明 消息的来源; 同时，消息 $m$ 的认证者 $T$ 也不能向第 3 方证明曾经向接收者 $R$ 提供了认证的消息 $m$, 该性质称为前向可否认性 ${ }^{[2]}$. 可否认性强化了安全协议的保密特性, 并在互联网密钥交换协议、 电子选举系统、电子商务系统等许多领域应用 ${ }^{[1-4]}$. 
可证明安全的可否认认证协议一直是该领域的重要研究内容. 为实现密码协议的安全性 证明, 通常采用Dolev和Yao ${ }^{[5]}$ 提出的协议参考模型描述安全协议. Dolev-Yao模型是一个独立 计算模型，该模型将安全协议抽象在一个隔离的环境中，并将协议简化为单一的协议参与方 集合, 协议参与方集合运行单一的协议实例. 基于Dolev-Yao模型和计算复杂性观点的安全定 义和分析方法, 在异步并行的网络环境中, 目前已提出了许多可证明安全的可否认认证协议. 例如，文献[1]提出了基于时间约束的可否认认证协议，该协议在认证过程中嵌套了时间延迟 的零知识证明协议. 文献 $[3,4]$ 分别提出了基于困难问题假设的可否认认证协议, 两个协议方 案在认证过程中假设存在一个协议双方都信任的公共目录. 最近, 文献[2]在BCK模型 ${ }^{[6]}$ 中利 用基于仿真的安全定义方法对可否认认证和前向可否认认证问题进行了统一的定义, 同时,提 出了基于多陷门承诺的可否认认证协议.

随着可证明安全方法学研究的逐步深入, 人们认识到协议的异步并发复合是现实网络环 境中协议运行的真实情景. 例如, 各种不同的密码协议是异步并发运行的; 协议参与方集合可 能不仅仅是一个, 参与方集合也可能存在交叉; 同一密码协议存在多个协议实例, 协议实例是 异步并发运行的. 为了更好地描述和分析异步并发环境下安全协议的并发复合问题, Canetti ${ }^{[7]}$ 提出了新的计算模型：通用可复合安全模型(universally composable，简称 UC).

利用UC安全模型研究可否认认证协议更符合文献[1]对并行可否认认证问题的定义. 基于 $\mathrm{UC}$ 安全框架的形式化方法可以单独设计协议, 只要协议满足UC 安全定义, 那么就能够保证 该协议和其他协议安全地并行复合运行. 在UC安全模型中, 利用“理想函数 $F$ ”对协议进行形 式化的安全定义. UC 安全是更高级别的安全定义, 它的抽象层次超过其他模型(例如Dolev-Yao 模型和BCK模型). UC安全模型最重要的属性是它能够确保协议在任意的和未知的多方环境中 运行时仍然是安全的, 因此它的安全定义更为严格.

“仿真”技术是可否认认证协议可证明安全的关键技术. 例如，如果接收者能够“独立仿真” 认证者和接收者之间的认证消息, 那么协议是可否认的; 如果认证者能够“独立仿真”自己的 内部状态信息以及向接收者提供的认证消息，那么协议是前向可否认的. 在 UC 安全模型中, 主要通过唯一会话标识 $S i d$ 和基于“混合模型 $\mathcal{F}$ ”的通用可复合定理解决协议异步并行消息的 交叉仿真问题. 在UC框架中, 假定 $n$ 个参与方 $P_{1}, \ldots, P_{n}$ 执行一个两方协议 $\pi$, 那么 $\left(P_{i}, P_{j}\right)$ 之间 运行的协议 $\pi$ 和 $\left(P_{i}, P_{j-1}\right)$ 之间运行的协议 $\pi$ 仅仅是不同的协议实例, 协议实例通过唯一的会话 标识 Sid区别. 为了描述真实场景协议的不同实例, 引入 “混合模型 $\mathcal{F}$ ”的概念, “混合模型 $\mathcal{F}$ ”简 化了通用可复合定理的描述, UC框架基于复合定理完成协议的复合安全证明, 协议并行运行 的消息复杂性是参与方线性相关的.

协议的综合效率与协议的结构密切相关. 可否认认证协议方案的结构主要分为 3 类. 例如, 基于零知识证明的CCA2 安全的加密体制结构的可否认认证协议(简称ZK-CCA2-based) ${ }^{[1,3,4]}$ 、

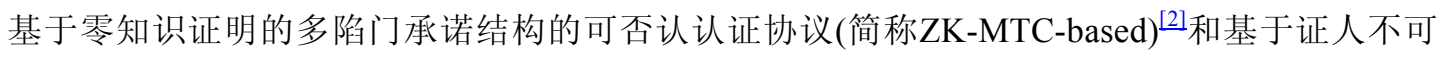
区分的不经意传输结构的可否认认证协议(简称WI-OT-based) ${ }^{[8]}$. 由于WI-OT-based结构的协议 利用了可验证平滑投影散列函数 ${ }^{[9]}$ (verifiably smooth projective hashing, 简称VSPH)和非承诺 加密体制 ${ }^{[10]}$ (non-committing encryptions, 简称NCE), 因此该结构也称为WI-VSPH-NCE- based 
结构. 文献[8]的工作是本文工作的基础.

本文与文献[8]的主要区别有两点：在理论层面，本文基于确定性复合剩余假设(decisional composite residuosity, 简称DCR $)^{[11]}$ 实现VSPH. 文献[8]协议方案的VSPH实现基于DDH假设. 针对带宽而言, DDH假设处理的消息是比特空间的, DCR假设可以处理的消息是指数空间的， 因此基于DCR假设实现的VSPH具有更高的计算效率. 在实现过程中，本文使用“与或”计算的 特性实现协议参与方共享秘密信息获取功能. 文献[8]的共享秘密信息获取利用了不经意传输 的思想, 为实现参与方共享秘密信息获取, 方案需要利用非承诺加密体制. 本文方案仅仅需要 使用VSPH，改善了协议的计算效率和通信效率，本文的协议结构简称WI-VSPH-based. 为了 实现前向可否认性, 本文修改了文献[9]提出的可验证平滑投影散列函数, 基于陷门承诺构造 了新的投影密钥函数, 修改的VSPH的安全性仍然可以归约为确定性复合剩余假设.

本文的安排如下: 第 1 部分介绍了UC安全模型和VSPH. 第 2 部分基于陷门承诺构造了新 的投影密钥函数, 建立了修改的VSPH. 第 3 部分定义了可否认认证协议的理想函数 $F_{\mathrm{CDA}}$. 第 4 部分提出一个新协议方案. 第 5 部分对新的可否认认证协议进行安全性分析, 证明了该协议安 全实现了理想函数 $F_{\mathrm{CDA}}$. 第 6 部分比较了相关工作. 第 7 部分是本文的结论.

\section{1 基础知识}

在介绍基础知识之前, 先描述两个与本文相关的定义. $k$ 是安全参数.

定义 1 (可忽略) 称函数 $F: \mathbb{N} \rightarrow \mathbb{N}$ 是可忽略的, 若对于任意的 $c \in \mathbb{N}$, 存在一个 $k_{0} \in \mathbb{N}$, 使得对 $k \geqslant k_{0}$ 有 $0 \leqslant F(k)<k^{-c}$.

定义 2 (计算不可区分性) 称 $X=\left\{X_{k}\right\}(k \in \mathbb{N})$ 和 $Y=\left\{Y_{k}\right\}(k \in \mathbb{N})$ 两个样本空间是多项式 时间不可区分的, 若对于任意概率多项式时间算法 $D$, 对于任意的 $c \in \mathbb{N}$, 存在一个 $k_{0} \in \mathbb{N}$, 使得对 $k \geqslant k_{0}$ 有 $\left|\operatorname{Pr}\left[D\left(X_{k}, \mathbb{N}\right)=1\right]-\operatorname{Pr}\left[D\left(Y_{k}, \mathbb{N}\right)=1\right]\right|<k^{-c}$ 是可忽略的.

\subsection{UC安全模型}

$\mathrm{UC}$ 框架为密码协议任务的安全定义提供了精确方法, 符合UC框架安全定义的协议称为 $\mathrm{UC}$ 安全的. 在 $\mathrm{UC}$ 框架中, 任何一个 $n$ 方协议 $\pi$ 都可以描述为一个交互式的概率多项式时间图 灵机系统, 该系统由 $n$ 个交互式图灵机 $P_{i}$ 组成, 其中 $0<i<n$. 一个交互式图灵机代表一个协议 参与方. 攻击者实体也被模型化为一个交互图灵机. 为描述协议异步并行运行的安全, UC框 架模型中有一个特殊的攻击者实体“环境机 $Z$ ”, $Z$ 以任意方式与攻击者及参与方交互. $Z$ 获得的 视图信息是协议的输出信息.

现实模型 攻击者 $A$ 与运行协议 $\pi$ 的参与方 $P_{1}, \ldots, P_{n}$ 共存. 假定所有参与方通过点对点通 信信道连接, 信道是公开的, 每一个参与方最初都是诚实的并且遵守预定的协议规则. 攻击者 可以在协议开始或在协议执行的任意点攻陷参与方. 一旦攻击者 $A$ 攻陷参与方, 攻击者 $A$ 将获 得参与方内部的状态数据. 令 $\operatorname{REAL}_{\pi, A, Z}(k, z, r)$ 表示当 $Z$ 与攻击者 $A$ 、参与方在运行协议 $\pi$ 时的 交互信息视图, 其中 $k$ 是安全参数, $z$ 是“环境机 $Z$ ”的输入信息, $r$ 是随机信息集合, $r=\left\{r_{Z}, r_{A}, r_{1}\right.$, $\left.r_{2}, \ldots, r_{n}\right\}$. 当 $r$ 均匀分布时, 分布空间 $X=\left\{\operatorname{REAL}_{\pi, A, Z}(k, z, r)\right\}_{k \in \mathbb{N}, Z \in\{0,1\}}$ *简记为 $\operatorname{REAL}_{\pi, A, Z}$. 
理想过程 理想过程包括理想函数 $F$, 理想过程攻击者 $S$ (也称为仿真器), 虚构的参与方 $p_{1}^{\prime}, \ldots, p_{n}^{\prime}$. 理想函数 $F$ 描述了协议的安全属性. 虚构的参与方之间只能借助理想函数 $F$ 在安全 信道中通信, 攻击者 $S$ 不能阅读安全信道的内容. 一旦虚构参与方得到某些输入, 它将该信息 传递给理想函数 $F$, 同时理想函数 $F$ 通过输出信息做出响应, 攻击者 $S$ 的能力与现实模型中 $A$ 一 样. 令 $\operatorname{IDEAL}_{F, S, Z}(k, z, r)$ 表示当 $Z$ 与攻击者 $S$, 理想函数 $F$ 运行时的交互信息视图, 参数 $k, z, r$ 定 义同上. 当 $r$ 均匀分布时, 分布空间 $Y=\left\{\operatorname{IDEAL}_{F, S, Z}(k, z, r)\right\}_{k \in \mathbb{N}}, Z \in\{0,1\}$ 简记为 $\operatorname{IDEAL}_{F, S, Z}$.

区分器 在理想过程和现实模型之间存在一个区分器 “ $Z$ ” $Z$ 通过提供参与方的输入, 阅 读参与方的输出与参与方交互, $Z$ 也可以单独与攻击者 $A$ 或 $S$ 通信.

定义 3 令 $n \in \mathbb{N}, F$ 是理想函数, $\pi$ 是 $n$ 方协议, 称 $\pi$ 安全实现 $F$, 若对于任何攻击者 $A$ 都存

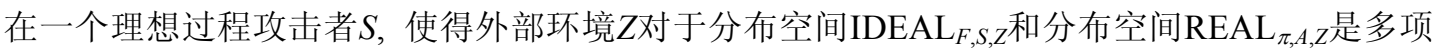
式时间计算不可区分的，并表示为

$$
\operatorname{REAL}_{\pi, A, Z} \approx \operatorname{IDEAL}_{F, S, Z} \text {. }
$$

为了实现协议的不同实例在并行复合情形下是安全的, UC 安全模型引入了混合模型 $\mathcal{F}$. 假定协议 $\rho$ 安全地 “实现”了理想函数 $F$, 那么称为混合模型 $\mathcal{F}$. 对于运行在混合模型 $\mathcal{F}$ 中的任 意协议 $\pi, \pi$ 协议主体可以以它希望的方式与理想函数 $F$ 的多个实例秘密通信, 不同实例通过 会话标识区分. 在混合模型 $\mathcal{F}$ 中的攻击者不能访问理想函数 $F$ 的内部实例消息. 外部环境 $Z$ 也不可以直接访问理想函数 $F$ 的内部实例消息. 协议的多个实例并行运行称为协议自复合.

定理 1 (自复合定理) $\pi$ 对理想函数 $F$ 实例的调用都转变为启动协议 $\rho$ 的实例, 同时协议

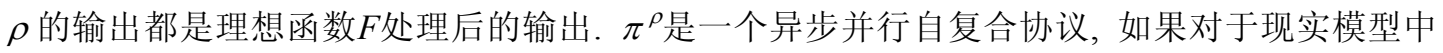
的攻击者 $A$, 不存在一个有效的“环境机” $Z$ 以不可忽略的概率分辨出是 $A$ 与现实模型中协议 $\pi^{\rho}$ 的交互信息视图, 还是 $S$ 与混合模型 $\mathcal{F}$ 中的 $\pi$ 协议的交互信息视图, 那么就说自复合协议 $\pi^{\rho}$ 安 全地实现了理想函数 $F$.

\section{2 可验证平滑投影散列函数}

Cramer和Shoup ${ }^{[12]}$ 首次定义了投影散列函数. 投影散列函数的定义依赖困难子集成员问 题. 困难子集成员问题针对每一个实例 $i$ 说明了两个有限非空集合 $X, W \subseteq\{0,1\}^{p l o y \mid k}$ 和一个 $\mathrm{NP}$ 关系 $\mathcal{R} \subset X \times W$, 即 $i=(X, W, \mathcal{R})$ 使得对应的语言 $L=\{x: \exists w . s . t(x, w) \in \mathcal{R}\}$ 是非空的, 试图区分 集合 $L$ 中的元素和集合 $X \mid L$ 的元素是困难的.

投影散列函数有两个密钥, 一个是秘密的散列密钥 $k$, 一个是公开的投影密钥 $\theta$. 投影散列 函数可表示为 $\boldsymbol{H}=\left(H_{k}, K, X, L, \Pi, \beta, \theta\right)$. 令 $\left\{H_{k}: X \rightarrow \Pi\right\}_{k \in K}$ 是散列函数集合, $X$ 是消息集合, $\Pi$ 是 散列函数值的集合, $K$ 是散列函数密钥集合, 投射密钥函数 $\beta: k \rightarrow \theta$, 即 $\theta=\beta(k), \theta$ 是投影密钥.

$\mathrm{VSPH}$ 整合了投影散列函数和可验证困难子集成员问题. VSPH强调了困难子集成员问题 采样的可验证性 ${ }^{[9]}$.

定义 4 可验证困难子集成员问题: 称困难子集成员问题 $M$ 是可验证样本，若下列条件成立:

1) 问题可采样: 存在一个概率多项式算法 $P(n), n=1^{k}$, 采样得到一个实例 $i=(X, W, \mathcal{R})$. 
2) 成员可采样: 存在一个输入是实例 $i=(X, W, \mathcal{R}) \in M$ 的概率多项式算法 $J$, 输出是 $x_{0} \in L$ 和 $w \in W$, 使得 $x_{0}$ 的分布是 $L$ 统计可忽略的均匀分布.

3) 非成员可采样: 存在一个输入是实例 $i=(X, W, \mathcal{R}) \in M$ 和元素 $x_{0} \in X$ 的概率多项式算法 $U$, 输出 $x_{1}=U\left(i, x_{0}\right)$ 使得如果 $x_{0} \in L$ 是均匀分布的, 那么 $x_{1}$ 的分布是 $X \backslash L$ 统计可忽略的均匀分布, 如果 $x_{0} \in X$, 那么 $x_{1}$ 的分布是 $X$ 统计可忽略的均匀分布.

4) 非成员可验证: 存在一个输入是任意的三元组 $\left(i, x_{0}, x_{1}\right)$ 的概率多项式算法 $V$, 可验证存 在比特 $b \in(0,1)$, 使得 $x_{1-b} \in X \backslash L$, 即

(a) 如果 $x_{0} \notin X \mid L$ 且 $x_{1} \notin X \backslash L$ 成立, 那么 $V\left(i, x_{0}, x_{1}\right)=0$;

(b) 存在比特 $b$, 如果 $x_{b} \in L, x_{1-b} \in U\left(i, x_{0}\right)$, 那么 $V\left(i, x_{0}, x_{1}\right)=1$.

定义 5 投影散列函数: 称 $(H, K, X, L, \Pi, \beta, \theta)$ 是困难子集成员问题 $M$ 的投影散列函数, 若 对每个实例 $i \in M$ 存在一个函数 $f$, 使得每一个 $x \in L(i)$ 和每一个 $k \in K(i), \theta=\beta(k)$, 有 $f(x, \beta(k), w)$ $=H_{k}(x)$.

定义 6 非成员平滑投影散列函数: 称 $(H, K, X, L, \Pi, \beta, \theta)$ 是困难子集成员问题 $M$ 的非成 员平滑投影散列函数, 若对每个实例 $i=(X, W, \mathcal{R})$ 和 $x_{1-b} \in X \backslash L,\left(\beta\left(k_{1-b}\right), H_{k}\left(x_{1-b}\right)\right)$ 和 $\left(\beta\left(k_{1-b}\right)\right.$, $\left.\Pi \leftarrow\{0,1\}^{k}\right)$ 是不可区分的.

\section{2 修改的VSPH}

确定性复合剩余假设 $[9,11]$ : 假设两个安全素数 $p$ 和 $q$ 满足 $p=2 p^{\prime}+1, q=2 q^{\prime}+1, p^{\prime}$ 和 $q^{\prime}$ 是素数, 令 $N=p q . J_{N^{2}}(1)$ 是 $Z_{N^{2}}^{*}$ 的子群, $J_{N^{2}}(1)=X=\left\{x \mid x \in Z_{N^{2}}^{*}, x / N=1\right\}$. 令 $L$ 是由 $J_{N^{2}}(1)$ 中的所有元 素 $T$ 次幂组成的子群, 其中 $T=N^{\lfloor\log N+1\rfloor}$. 仅仅已知 $N$, 区分 $X$ 和 $L$ 集合中的随机元素是困难的.

\section{1 基于确定性复合剩余假设的可验证子集成员问题 ${ }^{[9]}$}

1) 问题可采样.

(a) 产生两个随机 $k$ 比特安全参数的安全素数 $p$ 和 $q$, 令 $N=p q, N^{\prime}=p^{\prime} q^{\prime}, T=N^{\lfloor\log N+1\rfloor}$;

(b) 产生随机生成元 $g \in J_{N^{2}}$. 即, 随机选择元素 $\mu \in Z_{N^{2}}^{*}$, 令 $g=\mu^{2}\left(\bmod N^{2}\right)$;

(c) 输出实例 $i=(X, W, \mathcal{R})$, 其中 $X=J_{N^{2}}, L=\left\langle g^{T}\right\rangle, W=\{0,1, \ldots,\lfloor N / 2\rfloor\}, \mathcal{R}=\left\{\left(g^{T a}, a\right)\right.$ : $a \in W\}$.

2) 成员可采样: 随机选择 $a \in W$, 输出 $x_{0}=g^{T a} \in L(i)$.

3) 非成员可采样: 随机选择 $d \in\{1, \ldots, N-1\}, x_{1}=U\left(i, x_{0}, d\right)=x_{0}(1+d N)$, 即 $x_{1} \in X(i) \backslash L(i)$.

4) 非成员可验证: 根据输入 $\left(1^{k}, i, x_{0}, x_{1}\right)$, 存在比特 $b \in(0,1)$, 如果概率多项式算法 $V=1$, 当且仅当 $x_{0}, x_{1} \in J_{N^{2}}, x_{0} \neq x_{1},\left(x_{0} / x_{1}\right)^{N}=1\left(\bmod N^{2}\right)$.

\section{2 基于陷门承诺的投影密钥函数}

本文的投影密钥函数本质上是一个基于DCR假设构造的陷门承诺 ${ }^{[13]}$. 陷门承诺是指存在 一个抗碰撞函数, 对于抗碰撞函数, 假定不知道陷门信息, 对于不同的输入值, 通过函数发现 碰撞是不可能的; 如果已知陷门信息, 通过函数发现碰撞是容易的. 
密钥生成算法 $\mathcal{G}$ : 基于 DCR 假设, $\left(p k, s k \leftarrow \mathcal{G}\left(1^{k}\right), p k=(N, g), s k=\left(p^{\prime}, q^{\prime}\right), k\right.$ 是安全参数, 则 $\operatorname{ord}(X)=\lambda\left(N^{2}\right) / 2=p p^{\prime} q q^{\prime}=N \lambda(N) / 2$, 其中 $\lambda(N)=2 p^{\prime} q^{\prime}$.

投影密钥函数 $\beta$ : 随机选择密钥信息 $k \in Z_{N \lambda(N) / 2,}, r \in Z_{N}$, 并通过投影密钥函数 $\beta(r, k)=g^{k} \xi^{r}$ $\bmod N^{2}$ 提交投影密钥 $\theta$, 其中 $\xi=1+N \bmod N^{2}, \xi^{r}=1+r N \bmod N^{2}$.

定理 2 当投影密钥函数 $\beta(r, k)$ 的密钥信息是安全素数模因子, 投影密钥函数是符合陷门 承诺安全定义的.

证明 承诺的正确性: 对于任何 $r \in Z_{N}, k \in Z_{N \lambda(N) / 2}$, 由于 $1+r N$ 是 $X$ 中的元素, $g$ 是生成元, $g^{k}$ 在 $X$ 中是均匀分布的, 故 $\beta(r, k)=g^{k} \xi^{r} \in X$.

碰撞发现的正确性: 已知投影密钥 $\beta(r, k)\left(r \in Z_{N}, k \in Z_{N \lambda(N) / 2}\right)$ 和模的因子 $\left(p^{\prime}, q^{\prime}\right)$, 对于任 意 $r_{\beta} \in Z_{N}$, 可以容易地通过函数发现碰撞.

假设选择 $u$ 使得 $g^{\lambda(N)}=(1+u N) \bmod N^{2}$, 在 $Z^{*}{ }_{N}$ 中求出 $u$ 的逆元 $v$. 然后

$$
\beta(r, k)=g^{k} \xi^{r}=g^{k}(1+r N)=g^{k}(1+u v r N) \bmod N^{2}=g^{r+v r \lambda(N)} \bmod N^{2},
$$

则 $k_{\beta}=k+\left(r-r_{\beta}\right) d \lambda(N) \bmod N \lambda(N) / 2$.

安全性证明: 假设存在算法 $A$, 根据输入 $(N, g)$, 可以发现 $(k, r)$ 和 $\left(k_{\beta}, r_{\beta}\right)$, 使得 $\beta(r, k)=$ $\beta\left(k_{\beta}, r_{\beta}\right)$. 假设 $k \neq k_{\beta}$, 则 $g^{k}(1+r N)=g^{k \beta}\left(1+r_{\beta} N\right) \bmod N^{2}$, 然后令 $\Delta k=k_{\beta}-k, \Delta r=r-r_{\beta}$, 则 $g^{\Delta k}=$ $(1+\Delta r N) \bmod N^{2}$. 既然 $g$ 的阶为 $\lambda(N) N / 2,(1+\Delta r N)$ 的阶为 $N$, 这就意味 $\Delta r$ 是 $\lambda(N) / 2$ 的乘, 算法 $A$ 存 在意味着与因子分解困难问题假设矛盾.

\section{3 基于确定性复合剩余假设的可验证平滑投影散列函数}

对于 $i=(N, u) \in M$, 称 $(H, K, X, L, \Pi, \beta, \theta)$ 是基于确定性复合剩余假设的可验证平滑投影散 列函数, 如果下列定义成立:对于 $k \in Z_{N \lambda(N) / 2}$, 投影密钥算法 $\theta=\beta(r, k)=g^{k} \xi^{r} \bmod N^{2}=e \xi^{r} \bmod N^{2}$, $H_{k}(x)=x^{k} \xi^{\theta}\left(\bmod N^{2}\right)$, 其中 $e^{=} g^{k}, g=u^{2}\left(\bmod N^{2}\right), T=N^{\lfloor\log N+1\rfloor}, r \in Z_{N}, \xi=1+N \bmod N^{2}$.

定理 $3(H, K, X, L, \Pi, \beta, \theta)$ 是基于确定性复合剩余假设的可验证平滑投影散列函数.

证明 投影散列的有效性: 对于 $k \in Z_{N \lambda(N) / 2}$ 和 $x=g^{T a}\left(\bmod N^{2}\right) \in L$,

$$
H_{k}(x)=x^{k} \xi^{\theta}\left(\bmod N^{2}\right)=\left(g^{T a}\right)^{k} \xi^{\theta}\left(\bmod N^{2}\right)=\left(g^{k}\right)^{T a} \xi^{\theta}\left(\bmod N^{2}\right)=e^{T a} \xi^{\theta}\left(\bmod N^{2}\right) .
$$

非成员的可验证平滑投影散列函数: 假设 $i=(N, u)$, 令 $x_{1-b} \in X L$, 令 $k_{\beta}=k+\left(r-r_{\beta}\right) d \lambda(N) \bmod$ $N \lambda(N) / 2$, 由于每个 $\theta$ 都存在至少两个元素 $k, k_{\beta} \in K$ 使得 $\theta=\beta(r, k)=\beta\left(k_{\beta}, r_{\beta}\right)$, 同时由于 $x_{1-b} \neq$ $1\left(\bmod N^{2}\right), \theta$ 不能唯一地确定 $H_{k}\left(x_{1-b}\right)$, 即 $(H, K, X, L, \Pi, \beta, \theta)$ 是非成员的可验证的平滑投影散 列函数.

\section{3 可否认认证的安全模型}

\section{1 安全需求}

在对可否认认证协议的安全需求进行定义之前, 先对定义的描述符号进行说明. 参与方 为消息认证者 $T$ 和消息接收者 $R$; 消息认证码的随机密钥为 $k_{\mathrm{MAC}}$; 消息认证者 $T$ 需要认证的消息 为 $m$; 被认证消息的认证码为 $\mathrm{MAC}\left(m, k_{\mathrm{MAC}}\right)$; 消息认证者 $T$ 的秘密信息为 $S K_{T}$; 消息认证者 $T$ 的 公开信息为 $P K_{T}$; 安全的保密消息传输函数为 $\mathrm{SMT}\left(k_{\mathrm{MAC}}\right)$.

可否认认证协议的安全需求如下: 
（1）正确性：对任何消息 $m$, 如果认证者 $T$ 和接收者 $R$ 执行的协议是为了认证消息 $m$, 那 么，接收者 $R$ 接受.

（2）抗中间人攻击：假定认证者 $T$ 想要认证多项式数量的消息 $\left(m_{1}, m_{2}, \ldots\right)$, 这些消息可能 被自适应的攻击者 $A$ 自由地选择(例如, 假冒接收者 $\hat{R}$, 认证者 $T$ 提供预言机服务), 称攻击者 $A$ 成功地攻击了协议方案, 若存在一个伪造者 $C$ (被 $A$ 控制, 并且假冒成 $\hat{T}$ ), 成功地向接收者 $R$ 认 证了消息 $m \notin\left\{m_{1}, m_{2}, \ldots\right\}$. 抗中间人攻击保证所有的概率多项式时间的攻击者 $A$ 至多以可忽略 的概率成功.

(3) 可否认性：假定攻击者 $A$ 能力如上, 认证者 $T$ 将认证消息 $m$, 则对每一个攻击者 $A$, 都存 在一个多项式时间的可否认仿真器 $S_{\mathrm{CDA}}$, 仿真器的输出 $\operatorname{Sim}-\operatorname{Trans}\left(m, \operatorname{MAC}\left(m, k_{\mathrm{MAC}}\right)\right.$, $\left.\operatorname{SMT}\left(k_{\mathrm{MAC}}\right)\right)$ 与协议实际执行的输出 $\operatorname{Trans}\left(m, \operatorname{MAC}\left(m, k_{\mathrm{MAC}}\right), \operatorname{SMT}\left(k_{\mathrm{MAC}}\right)\right)$ 是不可区分的.

(4) 前向可否认性: 对于可否认认证协议, 认证者内部状态信息表示为 $\operatorname{Int}\left(m, S K_{T}\right.$, $\left.\operatorname{SMT}\left(k_{\mathrm{MAC}}\right)\right)$, 根据对消息 $m$ 的认证和秘密信息 $S K_{T}$ 存在一个多项式时间的前向可否认仿真器 $S_{\mathrm{F}-\mathrm{CDA}}$, 如果输入 $S K_{T}$ 和 $\operatorname{Trans}\left(m, \operatorname{MAC}\left(m, k_{\mathrm{MAC}}\right), \operatorname{SMT}\left(k_{\mathrm{MAC}}\right)\right)$, 仿真器 $S_{\mathrm{F}-\mathrm{CDA}}$ 对消息 $m$ 的认证存在 仿真的 $\operatorname{Sim}-\operatorname{Int}\left(m, S K_{T}, \operatorname{SMT}\left(k_{\mathrm{MAC}}\right)\right)$ 与真实的 $\operatorname{Int}\left(m, S K_{T}, \operatorname{SMT}\left(k_{\mathrm{MAC}}\right)\right)$ 是不可区分的.

(5) 并行复合安全: 使用可否认认证的系统几乎都总是高度并发的. 可否认认证协议将作 为许多应用中的子协议, 或者作为和其他应用复合在一起使用的协议. 在并发条件下攻击者 能自适应地修改通信内容，并发复合安全强调可否认认证和其他实体涉及协议是复合安全的.

本文考虑如下的安全假设：假定攻击者具有自适应攻击能力, 该攻击者在协议交互过程的 任何位置都可以攻陷参与方, 如果攻击者在攻陷某个协议参与方时可以获得参与方在协议执 行过程中使用的所有随机比特信息和秘密信息(内部状态数据), 那么称为 non-erase攻陷模型. 如果已知某些可信任的参与方建立的公开参考串, 那么称为公共参考串模型(common reference string, 简称CRS)模型 114,15$]$. 基于 CRS 模型, 本文在non-erase攻陷模型和自适应攻击者能力假 设下研究UC安全的可否认认证协议.

\section{2 可否认认证协议理想函数}

在UC安全框架中, 本文通过 “理想函数 $F_{\mathrm{CDA}}$ ”定义可否认认证协议的安全模型, 形式化的 可否认认证协议的理想函数 $F_{\mathrm{CDA}}$ 见图 1.

消息源注册 假定消息认证者需要认证消息，为了绑定认证消息，可以通过证人不可区 分(witness indistinguishable, WI) 或者零知识完成证明. 本文考虑使用 VSPH, 原因是 VSPH 具 有证人不可区分性质，接收者(攻击者)试图区分 NP 问题的成员采样和非成员采样是可忽略的.

共享信息获取 “消息认证码的随机密钥”是接收者和认证者共享的秘密信息, 接收者提 供秘密信息并安全传输秘密信息, 对于自适应攻击者, 安全传输秘密信息必须为仿真器提供 仿真机制. 一方面，接收者的认证密钥由接收者提供，协议具有了接收者可否认仿真性质. 另 一方面，消息源的证人不可区分表明没有攻击者可以获得证人区分信息. 假定理想函数仅仅 通过证人信息向认证者提供共享信息，一旦认证者被攻陷，那么证人信息暴露. 由于认证者获 得的共享信息来自接收者, 接收者不可否认协议消息. 为了解决该问题, 我们考虑如下:接收 
者通过引入辅助信息 $\theta$ 实现前向可否认, 认证者必须同时拥有辅助信息和证人信息才能获得 理想函数提供的共享秘密信息. 认证者对辅助信息是可仿真的, 接收者可以否认提供辅助信 息, 更进一步, 接收者否认曾经提供共享秘密信息, 最终解决了接收者可否认问题. 辅助信息 由两部分组成 $\theta=\theta_{b} \| \theta_{1-b}$, 其中 $b \in\{0,1\}, \|$ 表示串接. 认证者可选择辅助信息的一部分实现接 收者可否认问题证明.

假定协议参与方有 $n$ 个, 分别为 $P_{1}, \ldots, P_{n}$, 协议参与方与攻击者 $S$ 共存, 安全参数为 $k$, 证人关 系为 $R_{w}$. 消息认证者 $T$ 的秘密信息 $: S K_{T}$, 消息认证者 $T$ 的公开信息 $: P K_{T}$.

\section{消息源注册}

接收某个参与方 $T$ 的请求 (Identification, $S i d, P K_{T}$ ), 如果 $P K_{T}$ 不是首次公布信息, 那么忽略请 求. 否则, $P K_{T} \leftarrow \varphi \cup P K_{T}, \varphi$ 表示空集合，执行如下步骤:

1) 根据从 $T$ 接收到证人消息 $\left(w_{b}\right), b \in\{0,1\}$, 记录 $l i s t-w_{T} \leftarrow \varphi \cup\left\{w_{b}\right\}, \varphi$ 表示空集合，向攻击者 $S$ 和接收者 $R$ 发送 (Receipt, Sid).

2) 根据从 $T$ 接收到的任意消息 (Identification-prover, Sid, $\left.x_{b} ; x_{1-b}\right)$, 计算成员关系 $\mathcal{R}_{w}\left(x_{b}\right.$, list-w), 如果 $\mathcal{R}_{w}\left(x_{b}, l i s t-w\right)=1$ 那么向攻击者 $S$ 和接收者 $R$ 发送 (Identification-proof, Sid, $\left.x_{b}\right)$. 否则忽略后续请求.

\section{共享信息获取}

1) 根据从 $R$ 处接收到的消息 $\left(\operatorname{Send}, \operatorname{Sid}, T, R, \theta=\theta_{b} \| \theta_{b-1}\right)$, 假定 $x_{b} \in \mathcal{R}_{w}\left(x_{b}\right.$, list-w), $x_{1-b} \notin \mathcal{R}_{w}\left(x_{b}, l i s t-w\right)$, 向攻击者 $S$ 发送 $(S i d, \theta) . \theta$ 是辅助信息.

$2)$ 根据从 $R$ 处接收到的消息 $\left(S i d, k_{\mathrm{MAC}}\right)$ ，其中 $k_{\mathrm{MAC}} \leftarrow\left\{k_{\mathrm{MAC}-b}, k_{\mathrm{MAC}-1-b}\right\}$ ，记录 $\left(k_{\mathrm{MAC}-b}\right.$, $\left.k_{\mathrm{MAC}-1-b}\right)$.

3) 根据从 $T$ 处接收到的消息 $\left(\mathrm{Send}, \mathrm{Sid}, T, R, x_{b}, w, \theta_{b}\right)$, 如果 $\mathcal{R}_{w}\left(x_{b}, l i s t-w\right)=1$, 向 $T$ 提供 $(\mathrm{Sid}$, $\left.k_{\mathrm{MAC}-b}\right)$.

认证阶段

根据从 $T$ 接收到消息(Authentication, Sid, $\left.m, k_{\mathrm{MAC}-b}\right)$, 处理如下:

1) 根据从 $T$ 接收到(Authentication, $S i d, m)$, 如果 $m \in\{0,1\}^{*}$, 并存在 $\left(S i d, k_{\mathrm{MAC}-b}\right)$, 那么向 $S$ 发送( $\mathrm{Sid}, m, \mathrm{MAC})$, 其他情形忽略.

2) 根据 $T$ 的秘密信息: $S K_{T}$ 和 $\theta$, 以及 $R$ 提供的随机挑战信息 $\left(S i d, r_{\beta}\right)$, 获得与辅助信息相关 的可仿真内部状态信息 $k_{\beta}$, 向 $S$ 发送 $\left(S i d, k_{\beta}\right)$

验证阶段

根据从 $R$ 接收到的(MAC-verify, Sid, $m, \mathrm{MAC}, k_{\beta}, \theta$ ), 处理如下:

- 如果 $\left(T, \mathrm{MAC}, k_{\beta}, \theta\right)$ 可验证, 那么接收认证, 输出 $\mathrm{acc}=1$. 否则拒绝认证, 输出 $\mathrm{acc}=0$.

\section{图 1 可否认认证理想函数 $F_{\mathrm{CDA}}$}

认证阶段 认证者 $T$ 通过共享秘密信息提交认证消息. 同时，根据认证者 $T$ 的秘密信息 $S K_{T}$ 和接收者 $R$ 发出的随机挑战信息 $r_{\beta}$. 消息认证者 $T$ 计算 $k_{\beta}, k_{\beta}$ 消息与部分辅助信息 $\theta$ 相关, 是认证 者 $T$ 的可仿真验证信息. 
验证阶段 接收者 $R$ 验证 $k_{\beta}$ 消息的可仿真性. 然后根据共享秘密信息接受认证者的消息 认证.

定义 7 (UC安全的可否认认证协议) 令 $n \in \mathbb{N}, F_{\mathrm{CDA}}$ 是可否认协议理想函数, 假定协议 $\rho$ 安全地“实现”了理想函数 $F_{\mathrm{CDA}}, \pi$ 对理想函数 $F_{\mathrm{CDA}}$ 实例的调用都转变为启动协议 $\rho$ 的实例, 同 时协议 $\rho$ 的输出都是理想函数 $F_{\mathrm{CDA}}$ 处理后的输出. $\pi^{\rho}$ 是一个异步并行复合的可否认认证协议, 称 $\pi^{\rho}$ 是 $\mathrm{UC}$ 安全的, 如果对于现实模型中的攻击者 $A$, 不存在一个有效的“环境机” $Z$ 以不可忽略

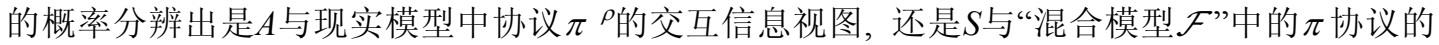
交互信息视图，那么协议 $\pi^{\rho}$ 安全地实现了理想函数 $F_{\mathrm{CDA}}$.

\section{4 可否认认证协议的实现}

文献[8]考虑协议的实现采用不经意传输协议的性质. 但是在可否认认证协议中利用UC安 全的不经意传输，必将降低协议的计算效率和通信效率。本文直接使用VSPH的性质和“与或 $\oplus$ ”计算的特性实现共享秘密信息获取功能，同时，令投影散列函数值 $H_{k}(x)=k_{\mathrm{MAC}}$ ，该方法满 足理想函数 $F_{\mathrm{CDA}}$ 的定义.

认证者进行采样, 接收者获得认证者提供的采样信息 $\left(x_{0}, x_{1}\right)$ 之后, 首先验证非成员采样成 立，然后随机选择投影散列函数的密钥信息，生成非成员投影散列函数值和成员投影散列函数 值，由于非成员平滑投影散列的随机分布和单向性，两个散列函数值通过 $\oplus$ 运算生成随机密文.

另外，接收者随机选择散列函数的密钥信息，利用投影密钥算法 $\beta$ 计算投影密钥 $\left(\theta_{b}, \theta_{1-b}\right)$. 如果投影密钥算法具有陷门承诺的性质, 一旦接收者提交投影密钥, 认证者可以根据成员采样 算法的秘密信息 $\left(p^{\prime}, q^{\prime}\right)$ 进行投影密钥的内部状态仿真, 即仿真接收者提供的散列函数密钥信息.

认证者利用证人信息、成员采样信息、接收者提供的投影密钥可以获得成员散列函数值, 然后获得非成员散列函数值, 即认证者获得秘密共享 $k_{\mathrm{MAC}}$. UC安全的可否认认证协议 UC-CDA的实现方案见图 2 .

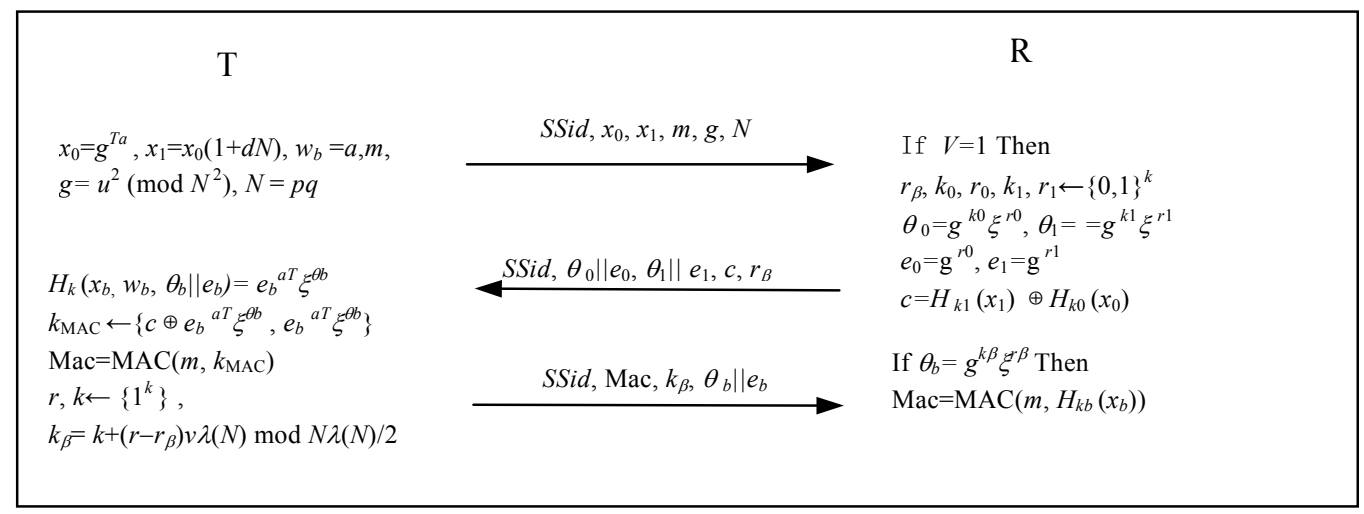

图 2 UC 安全的可否认认证协议

UC-CDA 协议的具体描述如下:

1) $T \rightarrow R$ : 认证者 $T$ 随机产生三元组 $\left(x_{0}, x_{1}, w_{b}\right)$, 其中 $x_{0} \in L,\left(x_{0}, w_{b}\right) \in \mathcal{R}, x_{1} \in X \backslash L$. 保留证人 
$w_{b} . x_{1}$ 由非子集成员算法 $U\left(i, x_{0}, d\right)$ 产生. 向 $R$ 发送 $\left(S S i d, x_{0}, x_{1}, N, g, m\right), S S i d$ 是UC-CDA协议并行 异步运行时一个协议实例的会话标识.

2) $R \rightarrow T$ : 根据接收到的消息 $\left(S S i d, x_{0}, x_{1}, N, g, m\right)$, 接收者 $R$ 执行非成员可验证算法 $V(\cdot)$. 如 果 $V(\cdot)=0$, 协议终止, 如果 $V(\cdot)=1$, 那么选择两个不同的随机散列函数密钥 $\left(k_{0}, k_{1}\right)$. 计算 $\left(H_{k 0}\left(x_{0}\right), H_{k 1}\left(x_{1}\right)\right)$, 执行投影密钥算法 $\beta(\cdot)$, 获得投影密钥 $\left(\theta_{0}, \theta_{1}\right)$. 计算 $\left(e_{0}, e_{1}\right), c=H_{k 0}\left(x_{0}\right) \oplus$ $H_{k 1}\left(x_{1}\right)$, 向 $T$ 发送 $\left\{S S i d, \theta_{0}\left\|e_{0}, \theta_{1}\right\| e_{1}, c, r_{\beta}\right\}$. 这里秘密共享信息 $k_{\mathrm{MAC}} \leftarrow\left\{H_{k 0}\left(x_{0}\right), H_{k 1}\left(x_{1}\right)\right\}$ 为可否 认认证协议的“消息认证码随机密钥”. 为了实现投影密钥的可否认验证, $r_{\beta}$ 为挑战信息.

3) $T \rightarrow R$ : 已知证人 $w_{b}$ 和投影密钥 $\theta_{b}$, 计算 $H_{k b}\left(x_{b}\right)=f\left(x_{b}, \theta_{b}, w_{b}\right), H_{k 1-b}\left(x_{1-b}\right)=c \oplus f\left(x_{b}, \theta_{b}, w_{b}\right)$. 获得 $k_{\mathrm{MAC}} \leftarrow\left\{H_{k 0}\left(x_{0}\right), H_{k 1}\left(x_{1}\right)\right\}$. 根据挑战信息 $r_{\beta}$ 和具有陷门承诺性质的投影密钥算法 $\beta(\cdot)$, 通过 $\theta \leftarrow\left\{\theta_{b}, \theta_{1-b}\right\}$ 随机绑定消息源秘密信息和消息认证码的密钥信息 $k_{\mathrm{MAC}}$, 计算认证者内部可仿真 状态信息 $k_{\beta}$. 最后计算认证消息的消息认证码 $\mathrm{Mac}=\mathrm{MAC}\left(m, k_{\mathrm{MAC}}\right)$. 向 $R$ 发送 $\left(S S i d, \theta_{b} \| e_{b}, k_{\beta}\right.$, Mac).

4) $R: \theta \leftarrow\left\{\theta_{b}, \theta_{1-b}\right\}$, 验证 $\theta_{b}=g^{k \beta} \xi^{r \beta}$ 是否成立, 如果成立, 由于 $R$ 知道 $k_{\mathrm{MAC}} \leftarrow\left\{H_{k 0}\left(x_{0}\right)\right.$, $\left.H_{k 1}\left(x_{1}\right)\right\}$, 验证 $\mathrm{Mac}=\mathrm{MAC}\left(m, k_{\mathrm{MAC}}\right)$ 是否成立.

\section{5 可否认认证协议的安全性分析}

根据 UC 安全的可否认认证协议的定义 7 , 在进行协议的安全性分析之前, 本文证明两个 引理.

引理 1 假定 VSPH 的投影密钥函数是陷门承诺安全的, 对于自适应攻击者, UC-CDA 协 议安全实现了前向可否认性.

证明 接收者利用保密信息 $\left\{k_{b}, k_{1-b}\right\}$ 提交投影密钥 $\left\{\theta_{b}, \theta_{1-b}\right\}$ 之后, 认证者根据投影密钥 $\left\{\theta_{b}, \theta_{1-b}\right\}$ 和成员采样证人信息 $w_{b}$ 可以获得投影散列函数值 $H_{k b}\left(x_{b}\right)=f\left(x_{b}, \theta_{b}, w_{b}\right)$ 并最终获得 $k_{\mathrm{MAC}}$ $\leftarrow\left\{H_{k 0}\left(x_{0}\right), H_{k 1}\left(x_{1}\right)\right\}$, 在协议执行结束后, 接收者希望对保密信息 $\left\{k_{\mathrm{b}}, k_{1-\mathrm{b}}\right\}$ 可否认, 即投影密钥 函数 $\beta(k, r)$ 应该提供可仿真机制.

由于认证者已知模因子, 根据定理 2 , 可以很容易得到 $k_{\beta}=k+\left(r-r_{\beta}\right) d \lambda(N) \bmod N \lambda(N) / 2$, 对于 接收者提供的投影密钥 $\left\{\theta_{b}, \theta_{1-b}\right\}$, 由于投影密钥可由认证者提供的 $\left(k_{\beta}, r_{\beta}\right)$ 验证, 对接收者提供 的 $(k, r)$ 可以仿真, 攻击者不能向第 3 方证明投影密钥以及秘密共享信息 $k_{\mathrm{MAC}}$ 与接收者相关, 即 前向可否认成立.

引理 2 基于CRS模型和可验证平滑投影散列函数, UC-CDA协议安全实现了理想函数 $F_{\mathrm{CDA}}$ 的共享信息 $k_{\mathrm{MAC}}$ 获取.

证明 $k_{\mathrm{MAC}}$ 共享信息获取通过成员采样实现. 首先使用可证明安全的仿真器技术, 建立 各种仿真情景, 然后证明 $\operatorname{IDEAL}_{(\mathrm{FCDA}, Z, S)} \approx \mathrm{REAL}_{(\mathrm{UC}-\mathrm{CDA}, Z, A)}$.

令 $A$ 是现实模型中的自适应攻击者, 现在构造一个理想过程中的攻击者 $S$ (称为仿真器 $S$ ). 在理想过程中, $S$ 可以与理想函数 $F_{\mathrm{CDA}}$ 及环境机 $Z$ 交互. $S$ 因 $A$ 的副本 $\tilde{A}$ 调用而工作, 副本 $\tilde{A}$ 与现 实模型中的 $A$ 交互. 对于仿真器 $S$, 在理想过程中的交互称为仿真器 $S$ 的外部交互, 与 $A$ 的副本 $\tilde{A}$ 之间的交互称为仿真器 $S$ 的内部交互. 基于公共参考串模型, 仿真器可以获得投影密钥 $\left\{\theta_{b}\right.$, $\left.\theta_{1-b}\right\}$ 和 $\left\{x_{b}, x_{1-b}\right\}$. 
仿真器 $S$ 通过 5 个具体的实现策略仿真了协议运行的全部状态, 仿真器 $S$ 的工作情形如下:

1) 仿真攻击者 $A$ 与 $Z$ 之间的通信: 仿真器 $S$ 将每一个从 $Z$ 接收到的输入信息写入副本 $\tilde{A}$ 的输入纸带(仿真 $A$ 获得在现实模型中来自于 $Z$ 的输入信息). 同样, $A$ 将写入自己输出纸带的 信息通过副本 $\tilde{A}$ 复制到 $S$ 的输出纸带(该信息被理想模型中的 $Z$ 阅读).

2) 仿真认证者和接收者未攻陷: 在理想过程中, 仿真器 $S$ 获得 SSid, 然后终止. 对于现实 模型中的认证者 $T$ 和接收者 $R$ 的仿真通过协议规程完成. 仿真器 $S$ 模拟虚拟的认证者 $T$ 随机生成 $\left(x_{0}, x_{1}, w_{b}\right)$. 模拟虚拟的接收者 $R$ 验证数据的非成员采样; 随机选择 $\left(k_{0}, k_{1}\right)$, 计算 $k_{\mathrm{MAC}} \leftarrow\{H$ $\left.{ }_{k b}\left(x_{b}\right), H_{k 1-b}\left(x_{1-b}\right)\right\}, c \leftarrow H_{k 1-b}\left(x_{1-b}\right) \oplus H_{k b}\left(x_{b}\right)$, 利用投影密钥算法 $\beta(\cdot)$ 生成投影密钥 $\left(\theta_{0}, \theta_{1}\right)$, 并发 送 $\left\{\theta_{0}\left\|e_{0}, \theta_{1}\right\| e_{1}, c, r_{\beta}\right\}$. 模拟虚拟的认证者 $T$ 接收 $\left\{\theta_{0}\left\|e_{0}, \theta_{1}\right\| e_{1}, c, r_{\beta}\right\}$ 并计算 $k_{\mathrm{MAC}} \leftarrow\left\{f\left(\theta_{b}\right.\right.$, $\left.\left.x_{b}, w_{b}\right), H_{k 1-b}\left(x_{1-b}\right)=c \oplus f\left(\theta_{b}, x_{b}, w_{b}\right)\right\}$. 由于两个参与方都没有被攻陷, 仿真器 $S$ 仿真的消息都是 随机分布的.

3) 仿真接收者和认证者都被攻陷: 由于协议参与方被 $A$ 攻陷, 仿真器 $S$ 使用副本 $\tilde{A}$ 提供 的协议参与方真实数据进行仿真.

4) 仿真认证者被攻陷, 接收者未攻陷: 仿真器 $S$ 在内部交互中获得副本 $\tilde{A}$ 攻陷认证者 $T$ 提 供的 $\left(x_{b}, x_{1-b}, b\right)$. 基于 $\mathrm{CRS}$ 模型, 仿真器 $S$ 在外部交互中利用投影密钥 $\left(\theta_{0}, \theta_{1}\right)$ (见 $F_{\mathrm{CDA}}$ 的共享信 息获取定义第 2 条)计算 $f\left(\theta_{b}, x_{b}, w_{b}\right)$, 随机产生 $H_{k 1-b}\left(x_{1-b}\right) \leftarrow\{0,1\}^{|k|}$, 获得 $c=H_{k 1-b}\left(x_{1-b}\right) \oplus f\left(\theta_{b}, x_{b}\right.$, $w_{b}$ ). 在内部交互中, 仿真器模仿接收者发送 $c$.

5) 仿真接收者被攻陷, 认证者未攻陷：基于CRS模型，仿真器 $S$ 在外部交互的仿真过程中 向理想函数 $F_{\mathrm{CDA}}$ 提交 $\left(x_{b}, x_{1-b}\right)$, 仿真器 $S$ 在内部交互中获得副本 $\tilde{A}$ 攻陷接收者 $R$ 提供的随机信息 $\left\{\theta_{0}\left\|e_{0}, \theta_{1}\right\| e_{1}, c, r_{\beta}\right\}$. 仿真器 $S$ 在外部交互的仿真过程获得 $k_{\beta}=k+\left(r-r_{\beta}\right) d \lambda(N) \bmod N \lambda(N) / 2$, 并模 仿接受者提交给理想函数 $F_{\mathrm{CDA}}$, 通过理想函数 $F_{\mathrm{CDA}}$ 获得 $k_{\mathrm{MAC}} \leftarrow\left\{H_{k \beta}\left(x_{b}\right), c \oplus H_{k \beta}\left(x_{b}\right)\right\}$ (见图 1 中 $F_{\mathrm{CDA}}$ 的共享信息获取定义). 在内部交互中, 仿真器仿真认证者 $T$ 获得 $k_{\mathrm{MAC}} \leftarrow\left\{H_{k \beta}\left(x_{b}\right)\right.$, $\left.c \oplus H_{k \beta}\left(x_{b}\right)\right\}$.

$\operatorname{IDEAL}_{(F, Z, S)} \approx \mathrm{REAL}_{(\mathrm{UC}-\mathrm{CDA}, Z, A)}$ 的证明. 由于 $Z$ 和 $A$ 及协议参与方交互获得的信息视图与理 想过程中 $Z$ 和 $S$ 及在内部交互中副本 $\tilde{A}$ 的信息视图等价, $Z$ 对理想过程和现实模型存在的信息视 图的不可区分性证明转化为仿真器 $S$ 的内部交互仿真与外部交互仿真的不可区分. 由于双方都 被攻陷和都未被攻陷的计算不可区分是直接的, 本文仅需证明单方被攻陷的不可区分. 仿真 器 $S$ 内部交互仿真与外部交互仿真的不可区分性最终归约为可验证平滑投影散列函数的安全 性.

1) 接收者 $R$ 被攻陷的仿真是不可区分的. 假定接收者 $R$ 被攻陷, 攻击者 $A$ 控制接收者 $R$, 攻 击者 $A$ 获得认证者 $T$ 的证人 $w_{b}$ 是可忽略的.

反证法. 假设存在一个不可忽略的概率多项式攻击算法 $B$ 预知 $w_{b}^{\prime}$. 攻击者 $B$ 可以通过预知 $w_{b}^{\prime}$ 进而区分 $x \in L$ 和 $x \in X$, 这将破坏困难子集成员问题 $M$ 的定义.

假定一个实例 $i(X, W, R) \leftarrow I_{k}$ 和一个元素 $x \in X$. (i ) 随机选择 $b$, 令 $x_{b}=x$. (ii) 利用算法 $\operatorname{NO}-\mathrm{M}(\cdot)$ 产生 $x_{1-b}$. (iii) 将 $\left(x_{0}, x_{1}\right)$ 发给攻击者 $B$, 并获得攻击者 $B$ 预知的 $w_{b}^{\prime}$. (iv) 如果 $w_{b}^{\prime}=w_{b}$ 那 么表明攻击者 $B$ 预知 $x \in L$. 如果 $w_{b}^{\prime} \neq w_{b}$, 那么表明攻击者 $B$ 预知 $x \in X$. 一方面, 如果预知 $x_{b} \in L$, 
那么存在一个不可忽略的概率多项式攻击者 $B$ 预知 $w_{\mathrm{b}}$, 与本文定义 4 矛盾. 另一方面, 如果 $x_{b}$ $\in X$, 那么 $x_{1-b} \in X$, 这种情形 $w_{b}$ 是不可预知的(信息论已有理论). 既然不存在不可忽略的概率 多项式攻击者 $B$, 那么也不可能存在同样能力的攻击者 $A$ 和 $Z$.

2) 认证者 $T$ 被攻陷的仿真是不可区分的. 假定认证者 $T$ 被攻陷, 攻击者 $A$ 控制认证者 $T$, 攻 击者 $A$ 对随机分布的密文信息的可区分是可忽略的. 根据非成员采样, 必存在 $x_{b} \in X \backslash L$ 或 $x_{1-b} \in X \backslash L, b \in\{0,1\}$, 否则, 协议终止. 不失一般性, 假定 $x_{1-b} \in X \backslash L$, 那么根据可验证平滑投影 散列函数的性质(见本文定义 6$),\left[\theta\left(k_{1-b}\right), H_{k 1-b}\left(x_{1-b}\right)\right]$ 和 $\left(\theta\left(k_{1-b}\right), r \leftarrow\{0,1\}^{|k|}\right)$ 是不可区分的, 从 而 $\left[\theta\left(k_{1-b}\right), c \leftarrow H_{k 1-b}\left(x_{1-b}\right) \oplus H_{k b}\left(x_{b}\right)\right]$ 与 $\left[\theta\left(k_{1-b}\right), c \leftarrow\{0,1\}^{|k|}\right]$ 是不可区分的均匀分布. 另外, 根据 投影密钥函数的性质, $\left[\theta\left(k_{1-b}\right), c \leftarrow H_{k 1-b}\left(x_{1-b}\right) \oplus H_{k \beta}\left(x_{b}\right)\right]$ 和 $\left[\theta\left(k_{1-b}\right), c \leftarrow H_{k 1-b}\left(x_{1-b}\right) \oplus H_{k b}\left(x_{b}\right)\right]$ 是不 可区分的,

3) 基于non-erase模型的仿真是不可区分. 仿真器 $S$ 在仿真过程中需要仿真参与方的随机 信息并写入随机纸带, 有些随机信息是参与方的内部数据, 例如, $k_{b}, r_{b}, c=H_{k 1-b}\left(x_{1-b}\right) \oplus f\left(\theta_{b}\right.$, $\left.x_{b}, w_{b}\right)$. 本协议通过可验证困难子集采样和投影散列算法的性质保证随机信息的均匀分布, $Z$ 试 图区分均匀分布的随机信息是可忽略的.

因此共享信息 $k_{\mathrm{MAC}}$ 获取是 $\operatorname{IDEAL}_{(F, Z, S)} \approx \mathrm{REAL}_{(\mathrm{UC}-\mathrm{CDA}, Z, A)}$.

定理 4 基于CRS模型, 可否认认证协议UC-CDA安全实现理想函数 $F_{\mathrm{CDA}}$.

证明 首先证明正确性、抗中间人攻击、协议可否认性, 然后证明协议是 UC 安全的. 正确性：根据协议规则，协议实施了认证方对消息 $m$ 的认证，正确性成立.

抗中间人攻击：1）根据 VSPH 的构造，成员采样和非成员采样的证人信息是不可区分的. 如果存在攻击者区分证人消息, 那么破坏困难子集成员问题 $M$ 的定义. 根据引理 2 , 攻击者试 图获得证人的消息是可忽略的, 认证方获得共享秘密. 2) 根据 VSPH 的投影密钥算法 $\theta=\beta(k$, $r$ ), 如果存在攻击者伪造 $\theta$, 与定理 2 矛盾, 同时 VSPH 是不安全的. 3) 接收者通过共享秘密验 证了消息认证.

可否认性：1) 根据引理 1 , 接收者 $R$ 提供了投影密钥信息, 认证者获得共享信息 $\left(k_{\mathrm{MAC}}\right)$, 同 时投影密钥通过认证者已知模因子可仿真, 接收者是可否认的(前向可否认性). 2) 根据引理 2, 接收者 $R$ 向认证者 $T$ 提供共享信息 $\left(k_{\mathrm{MAC}}\right)$, 认证者使用共享信息 $\left(k_{\mathrm{MAC}}\right)$ 进行消息认证, 认证者是 可否认的. 协议安全实现了完全的可否认性.

协议是自适应攻击者UC安全的：仿真器 $S$ 对协议做高层仿真, 详细细节与引理 2 证明相似. 认证者被攻陷. 仿真器获得认证者秘密信息, 根据引理 2 , 接收者的密文 $c$ 可仿真. 根据引理 1 , 接收者提供的投影密钥可仿真. 其他随机信息是均匀分布的. 接收者被攻陷. 仿真器获得接收 者秘密信息, 根据引理 2 , 认证者对获取共享信息 $\left(k_{\mathrm{MAC}}\right)$ 可仿真. 仿真器 $S$ 内部交互仿真与外部 交互仿真的不可区分性由引理 1 和 2 成立.

推论 1 在混合模型 $\mathcal{F}$ 中, 并行可否认认证协议 UC-CDA 是 UC 安全的.

证明 根据定理 1 (复合定理)和 4 及定义 7 , 推论得证.

\section{6 相关工作比较}


表 1 将本文方案与其他代表性方案进行了比较, 包括建立假设、协议结构、安全分析模型、 攻击者能力、计算复杂性、通信复杂性和前向可否认性.

\section{表 1 不同方案的比较}

\begin{tabular}{cccccc}
\hline & Dwork $^{[1]}$ & Deng ${ }^{[4]}$ & Raimondo $^{[2]}$ & 冯涛和马建峰 & 本文 \\
\hline 建立假设 & 时间约束 & 公共目录 & 时间约束 & 公共参考串 & 公共参考串 \\
协议结构 & $\mathrm{CCA}+\mathrm{ZK}$ & $\mathrm{CCA}+\mathrm{Hash}+\mathrm{ZK}$ & $\mathrm{MTC}+\mathrm{ZK}$ & $\mathrm{VSPH}+\mathrm{NCE}+\mathrm{WI}$ & $\mathrm{VSPH}+\mathrm{WI}$ \\
安全分析模型 & sequence concurrent & stand-alone & BCK Security & $\begin{array}{c}\text { UC Security } \\
\text { adaptive }+\end{array}$ & UC Security \\
攻击者能力模型 & active + erase & active + erase & active+non-erase & adaptive + non-erase \\
计算复杂性 & $O(2 m / 3)$ & $O(m / \mid$ hash $\mid)$ & $O(m / c)$ & $O(1)$ & $O(1)$ \\
通信复杂性 & $2 m+8$ & $11 m /|h a s h|$ & $20 m / c$ & $18 / m$ & $12 / m$ \\
前向可否认性 & 是, 未证明 & 不是 & 是, 可证明 & 是, 可证明 & 是, 可证明 \\
\hline
\end{tabular}

通信复杂性说明. 假定协议认证消息的长度为 $|m|$, Aumann 和 Rabin 方案是按 1 bit 位可否 认认证的, 通信复杂度为 $O(m)$, Deng 方案将认证消息分为 $t$ 块, 每块处理 hash-bit 位, 通信复 杂度为 $O(m / \mid$ hash $\mid)$, Raimondo 方案可否认认证的消息 bit 位与多陷门承诺(MTC)的消息空间密 切相关, 假定 MTC 的消息处理空间为 c-bit 位, 那么通信复杂度为 $O(m / c)$. Dowork 方案和本文 方案都使用了接收者提供的共享秘密信息, 但是 Dowork 方案利用非交互零知识证明(NIZK) 实现并行的不可伪造性, NIZK 的消息处理空间为 1-bit 位, NIZK 执行了 (2/3) $m$ 次的, 通信复杂 度为 $O(2 \mathrm{~m} / 3)$. Feng-Ma方案和本文方案为了实现高通信效率, 都利用 VSPH 实现了证人不可区 分和共享秘密信息传输, 通过一次协议执行完成可否认认证, 通信复杂度为 $O(1)$.

计算复杂性说明. 假定一次模指数计算为单位 1 , 那么零知识证明的复杂度为 $|Z K|=3^{[4]}$, CCA2 公钥加密复杂度为 $|E|=5$, 解密复杂度为 $|D|=3^{[12]}$; 多陷门承诺协议复杂度为 $|\mathrm{MTC}|=10^{[2]}$. 可验证平滑投影散列的复杂度为 $|\mathrm{VSPH}|=9^{[8]}$, 非承诺加密/解密 $|\mathrm{NCE} / \mathrm{NCD}|=6^{[8]}$. 本文方案与其他 代表性方案的计算复杂性见表 1 .

\section{7 结论}

本文研究了交互式的 CDA协议, 形式化的定义了 $\mathrm{UC}$ 框架的理想函数 $F_{\mathrm{CDA}}$, 在non-erase攻 陷模型的安全假设下，解决了异步并行环境中抗自适应攻击者能力的可否认认证协议问题. 基于陷门承诺构造了新的投影密钥函数和可验证平滑投影散列函数，协议的安全性可以归约 为确定性复合剩余假设，改善了协议的计算效率和通信效率. 因此实用性更好.

\section{参考文献}

1 Dwork C, Naor M, Sahai A. Concurrent zero-knowledge. In: Vitter J, ed. Proceedings of the 13th Annual ACM Symposium on Theory of Computing. New York: ACM Press, 1998. 409-418

2 Raimondo M d, Gennaro R. New approaches for deniable authentication. In: Proceedings of the 12th ACM Conference on Computer and Communications Security. New York: ACM Press, 2005. 112-121

3 Aumann Y, Rabin M. Authentication enhanced security and error correcting codes (extended abstract). In: Pro- 
ceedings of the 18th Annual International Cryptology Conference on Advances in Cryptology. London: Springer-Verlag, 1998. 299-303

4 Deng X, Lee C H, Zhu H. Deniable authentication protocols. IEE Proc Comput Digit Tech, 2001, 148(2): 101104 [DOI]

5 Dolev D, Yao A. On the security of public key protocols. IEEE Trans Inf Theor, 1983, 29(2):198-208 [DOI]

6 Bellare M, Canetti R, Krawzyk H. A modular approach to the design and analysis of authentication and key exchange protocols. In: Proceedings of the 30th Symposium on Theory of Computing. Dallas: ACM Press, 1998. $419-428$

7 Canetti R. Universally composable security: a new paradigm for cryptographic protocols. In: Proceedings of the 42nd IEEE Symposium on FOCS. New York: IEEE Computer Society Press, 2001. 136-145

8 冯涛, 马建峰. 基于证人不可区分的通用可复合安全并行可否认认证. 软件学报, 2007, 18(11): 2871一 2881

9 Kalai Y T. Smooth projective hashing and two-message oblivious transfer. In: Advances in Cryptology-Euro Crypt. 2005. LNCS 3494. Berlin: Springer-Verlag, 2005. 78-95

10 Damgard I, Nielsen J B. Improved non-committing encryption schemes based on general complexity assumptions. In: CRYPTO' 00. LNCS 1880. Berlin: Springer-Verlag, 2000. 432-450

11 Paillier P. Public-key cryptosystems based on composite-degree residuosity classes. In: Advances in Cryptology, Euro Crypt' 99. LNCS 1592. Berlin: Springer-Verlag, 1999. 223-238

12 Cramer R, Shoup V. Universal Hash proofs and a paradigm for adaptive chosen cipher text secure public-key encryption. In: Advances in Cryptology, Euro Crypt 2002. LNCS 2332. Berlin: Springer-Verlag, 2002. 45-64

13 Bresson E, Catalano D, Pointcheval D. A simple public-key cryptosystem with a double trapdoor decryption mechanism and its applications. In: Laih C S, ed. Advances in Cryptology Proceedings of Asiacrypt' 03. LNCS 2894. Berlin: Springer-Verlag, 2003. 37-54

14 Blum M, Feldman P, Micali S. Non-Interactive zero-knowledge and its applications. In: Proc 20th STOC. New York: ACM Press, 1988. 103-112

15 Zhang F, Ma J F, Moon S J. Universally composable anonymous Hash certification model. Sci China Ser F-Inf Sci, 2007, 50(3): 440-455 [DOI] 